\title{
Shish kebabs; hydrodynamic factors affecting their crystal growth
}

\author{
M. R. Mackley \\ With 2 figures and 1 table
}

(Received May 14, 1974)

\section{Introduction}

Hydrodynamic factors affecting the crystal growth of shish kebabs once nucleated and anchored in a uniformly flowing polymer stream are examined and the results discussed in terms of a general mechanism by which shish kebabs can grow.

Recent experiments of Pennings, Zwijnenburg and Lageveen (1) have shown that bundles of shish kebab type crystals which have been nucleated by extensional or turbulent flow, will, when anchored in a simple shear velocity gradient grow at the free end even at rather low velocity gradients. Prior to this experiment it has been thought necessary to nucleate and grow polyethylene shish kebabs, of the form shown in Figure (1) either in turbulent flow (2) or in a flow field containing a longitudinal velocity gradient (extensional flow) (3, 4 ), as these flow fields were thought neces-

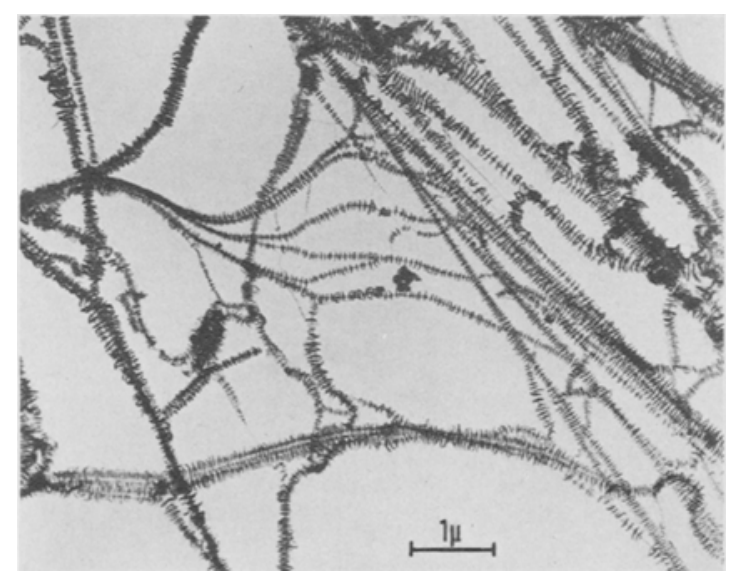

Fig. 1. Electron micrograph of typical polyethylene shish kebabs grown and thoroughly washed at $T=$ $103^{\circ} \mathrm{C}$ sary to initially elongate the molecules prior to crystallization, enabling the chain extended backbone to form. Earlier work of Pennings (3) supported by theoretical predictions $(5,6)$ found that simple shear alone had insufficient chain extending ability to nucleate shish kebab growth from polymer solution.

The result that shish kebabs, once nucleated and anchored can grow in a simple shear velocity gradient is at first surprising, however, closer examination of the hydrodynamic flow field experienced by the growing fibre reveals that the nature of the local flow field around the fibre may control growth to a far greater extent than the externally applied velocity gradient.

\section{Mathematical Formulation}

We will consider the problem of uniform streaming around an anchored fibre lying parallel to the stream direction. This situation is not identical to Pennings' experiment as in this case the fibres were anchored in a weak simple shear velocity gradient $\left(0.7 \mathrm{sec}^{-1}\right)$; however it will be shown that as far as local velocity gradients around the fibre are concerned the two situations are essentially identical. The same case of uniform streaming parallel to the fibre axis also occurs to a first approximation in the typical solution growth case of shish kebab fibres attached either to a rotating paddle or cylinder.

In formulating a model system it is necessary to make several simplifying assumptions. We will consider the flow around a single backbone and assume that the flow field is independent of neighbouring fibres; also we assume that the backbone is the only com- 
ponent of the shish kebab grown during flow and that the platelet component of the shish kebab is a type of chain folded epitaxial growth which occurs after the backbone has formed.

From electron micrographs of the type shown in Figure (1) we note that the backbone of the fibre is limited in all cases to a maximum diameter of $\sim 200 \AA$ and the fibre can be of indefinite length. Pennings (7) also observed that the fibres tapered smoothly towards the tip of the few fibre ends he did observe. The degree of rigidity of the fibre is not known explicitly; in the following analysis for mathematical simplicity it will be assumed to be rigid. Estimates of the displacement of the fibre by Brownian motion using Einstein's equation (8) suggest that this assumption is reasonable for the time scales involved in this problem.

We will assume the fibre approximates to a rigid prolate ellipsoid of high aspect ratio. The diameter of the ellipsoid is fixed at the experimentally observed value of $200 \AA$.

The choice of a suitable length is complicated by the requirement that the ellipsoid represents a realistic thickness of the fibre at the tip. For an ellipsoid with semi minor axis $b=10^{-6} \mathrm{~cm}$ and semi major axis $a=$ $10^{-4} \mathrm{~cm}$ the thickness of the fibre $10 \AA$ back from the tip is $12.6 \AA$ which appears realistic. Unfortunately larger values of $a$, which give more realistic experimentally observed values for the fibre length give increasingly unrealistic tip dimensions. In the analysis to follow, two ellipsoids will be considered both with $b=10^{-6} \mathrm{~cm}$ but in one case $a=1 \mathrm{~cm}$ and in the other $a=10^{-4} \mathrm{~cm}$. When discussing the results it will be born in mind that when $a=1 \mathrm{~cm}$ the tip dimensions are unrealistic.

In the real situation the fibre is anchored in some way at one end in the stream. The fibre will then take up an orientation such that the fibre axis is downstream and parallel to the direction of flow. By analogy we assume the major axis of the prolate ellipsoid to be oriented parallel to the flow and stationary with respect to the stream flow. Fig. 2 is a diagrammatic picture of the flow where the $z$ axis is parallel to the major axis and $\varrho$ corresponds to the radial distance from this axis.

In order to analyse the flow pattern around the ellipsoid it is necessary to know the Reynolds number $R$, relevant parameters are:

$$
\begin{aligned}
& \text { Viscosity } \quad \eta \sim 1 \text { poise (assuming Newtonian } \\
& \text { How) } \\
& \text { Density } \quad D \sim 1 \mathrm{grm} / \mathrm{cc} \\
& \text { Stream velocity } u=10 \mathrm{~cm} / \mathrm{sec} \\
& \text { Typical dimension } \\
& \text { for short fibre } \quad d=\text { length of fibre } 2 a=2 \cdot 10^{-4} \mathrm{~cm} \\
& =\text { diameter of fibre } 2 b=2.10^{-6} \\
& \mathrm{~cm}
\end{aligned}
$$

Now $R=\frac{D u d}{\eta}$.

If we choose as a typical dimension the length of the fibre $R=2.10^{-3}$ and if we choose the diameter $R=2 \cdot 10^{-5}$. For both cases a 'Stokes flow' solution is quite appropriate where all inertia terms may be ignored. If we

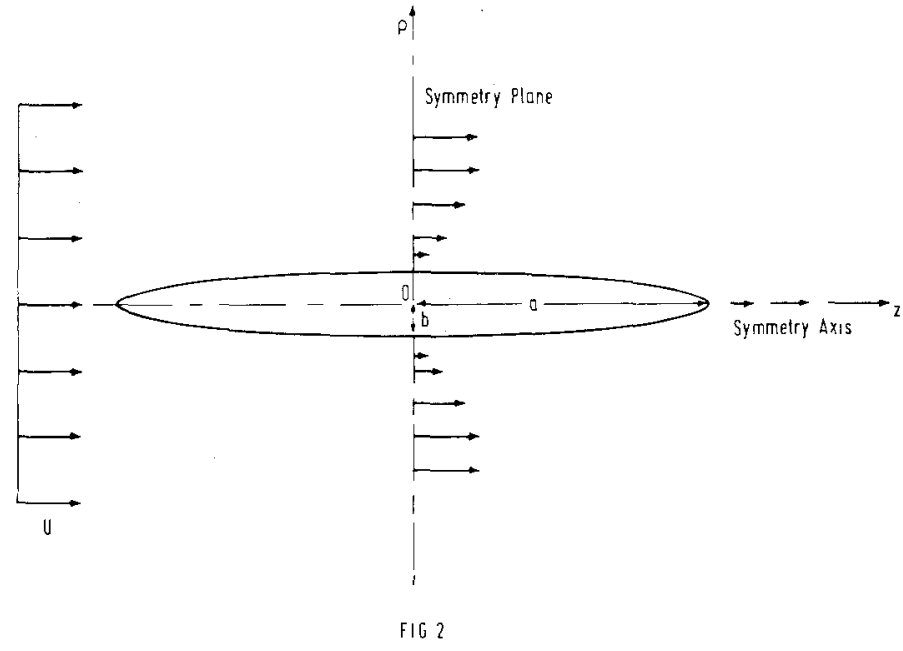

Fig. 2. Schematic diagram illustrating the nature of flow around a prolate ellipsoid 
choose the length of the 'long fibre' as a typical dimension the Reynolds number is 20 , which is moving out of the Stokes flow regime; however because the $b$ dimension is very small it was felt justifiable to continue in examining the Stokes flow solution.

\section{Stokes flow solution}

Simple symmetry considerations for Stokes flow around an ellipsoid illustrate some important points. With reference to Fig. 2, the flow around the ellipsoid must be symmetrical about the symmetry plane and possess rotational symmetry about the symmetry axis, also the velocity must be zero at all points on the surface of the ellipsoid. From these criteria we can conclude that immediately downstream of the ellipsoid tip along the symmetry axis $o z$.

$$
\dot{\gamma}_{L}=\frac{\delta V_{z}}{\delta z}>0 \text { and } \dot{\gamma}_{T}=\frac{\delta V_{z}}{\delta \varrho}=0
$$

That is, along the symmetry axis the longitudinal velocity gradient $\dot{\gamma}_{L}$ is finite and positive and the transverse velocity gradient $\dot{\gamma}_{T}$ is zero.

In a similar way on the symmetry plane

$\dot{\gamma}_{L}=\frac{\delta V_{z}}{\delta z}=0$ and $\dot{\gamma}_{T}=\frac{\delta V_{z}}{\delta \varrho}>0$.

That is, on the symmetry plane the transverse velocity gradient is finite and positive and the longitudinal velocity gradient zero. In order to determine the magnitude and extent of these velocity gradients it is necessary to solve the Stokes flow equations for this case.

The Stokes flow stream function around a prolate ellipsoid with major axis parallel to the direction of flow is given by Happel and Brenner (9). For the case where the ellipsoid is stationary with respect to the flow the stream function has the form:

$$
\begin{aligned}
\psi= & \frac{1}{2} u \varrho^{2} \\
& {\left[1-\left\{\frac{\left(\tau_{0}^{2}+1\right) \operatorname{coth}^{-1} \tau-\tau \frac{\left(\tau_{0}^{2}-1\right)}{\left(\tau^{2}-1\right)}}{\left(\tau_{0}^{2}+1\right) \operatorname{coth}^{-1} \tau_{0}-\tau_{0}}\right)\right] }
\end{aligned}
$$

where; $u=$ velocity of uniform stream flow.

$a=$ length of semi major axis of ellipsoid.

$b=$ length of semi minor axis of ellipsoid.

$\tau=\cosh \varepsilon \quad \tau_{0}=\cosh \varepsilon_{0}=\frac{a}{\sqrt{a^{2}-b^{2}}}$

$\varepsilon$ and $\eta$ are prolate spheroidal coordinates related to $z$ and $\varrho$ by;

$z=\left(a^{2}-b^{2}\right)^{\frac{1}{2}} \cosh \varepsilon \cos \eta$

$\varrho=\left(a^{2}-b^{2}\right)^{\frac{1}{2}} \sinh \varepsilon \sin \eta$

By suitable differentiation of equation [1] indicated in reference (9) we can obtain the velocity at various points around the ellipsoid. This gives the velocity $V_{z}$ along the symmetry axis where $\eta=0$ as;

$$
\begin{aligned}
& \left(V_{z}\right)_{\eta=0}=\frac{1}{\left(a^{2}-\overline{b^{2}}\right)^{\frac{1}{2}} \varrho \sin \eta} \cdot \frac{\delta \psi}{\delta \eta} \\
& \left(V_{z}\right)_{\eta=0}=u \\
& {\left[\begin{array}{l}
\left(\tau_{0}^{2}+1\right) \operatorname{coth}^{-1} \tau-\tau \frac{\left(\tau_{0}^{2}-1\right)}{\left(\tau^{2}-1\right)} \\
\left(\tau_{0}^{2}-1\right) \operatorname{coth}^{-1} \tau_{0}
\end{array}\right] .}
\end{aligned}
$$

For the particular case under consideration $a \gg b$. This enables us to make the approximation;

$$
\tau=\frac{z}{\left(a^{2}-b^{2}\right)^{\frac{1}{2}}} \sim \frac{z}{a}\left[1+\frac{1}{2}\left(\frac{b}{a}\right)^{2}\right]
$$

and

$$
\tau_{0}=\frac{a}{\left(a^{2}-b^{2}\right)^{\frac{1}{2}}} \sim 1+\frac{1}{2}\left(\frac{b}{a}\right)^{2} .
$$

Considering two cases;

\section{Case 1}

Let $z=n a$. Where $n>1$, then using the above approximations, substituting in [2] gives the approximate solution.

$$
\begin{array}{r}
\left(V_{z}\right)_{\eta_{0}} \approx u\left[\begin{array}{r}
\ln \frac{(n+1)}{(n-1)} \\
2 \ln 2\left(\frac{a}{b}\right)-1
\end{array}\right] \quad[3] \\
n>1 \quad \frac{b}{a} \ll 1
\end{array}
$$

Case 2

Let $z=a+x$. Where $x \ll a$. 
Then

Substituting in [2] gives

$$
\left(V_{z}\right)_{\eta_{0}}=u\left[1-\frac{\ln \frac{2 a}{x}}{2 \ln 2\left(\frac{a}{b}\right)-1}\right]
$$

$$
x \ll a \quad\left(\frac{a}{b}\right)^{2} \gg \frac{a}{x}
$$

Equations [3] and [4] give the velocity at different positions along the symmetry axis. The results for an ellipsoid where $a=1$ and $10^{-4} \mathrm{~cm}, b=10^{-6} \mathrm{~cm}$ is given in Table 1 .

The velocity in the $z$ direction along the symmetry plane where $\eta=\frac{\pi}{2}$ can be found in a similar way and is given by;

$$
\begin{aligned}
& \left(V_{z}\right)_{\eta=\frac{\pi}{2}}=\frac{1}{\left(a^{2}-b^{2}\right)^{\frac{1}{2}} \varrho \cosh \varepsilon} \frac{\delta \psi}{\delta \varepsilon} \\
& \left(V_{z}\right)_{\eta=\frac{\pi}{2}}=u \\
& {\left[1-\left(\frac{\left(\tau_{0}^{2}+1\right) \operatorname{coth}^{-1} \tau-\frac{\tau_{0}^{2}}{\tau}}{\left(\tau_{0}^{2}+1\right) \operatorname{coth}^{-1} \tau_{0}-\tau_{0}}\right)\right]}
\end{aligned}
$$

in this case; $\tau=\left(1+\left(\frac{\varrho}{a}\right)^{2}\right)^{\frac{1}{2}} \sim 1+\frac{1}{2}\left(\frac{\varrho}{a}\right)^{2}$

where $a \gg \varrho$

$\tau_{0} \sim 1 \frac{1}{2}\left(\frac{b}{a}\right)^{2}$

where $\left(\frac{b}{a}\right) \ll 1$.

Similarly approximate solutions are;

$$
\begin{aligned}
& \left(V_{z}\right)_{\eta=\frac{\pi}{2}}=u\left[1-\left\{\frac{2 \ln 2\left(\frac{a}{\varrho}\right)-1}{2 \ln 2\left(\frac{a}{b}\right)-1}\right\}\right] \quad[6] \\
& a \gg \varrho \quad a \gg b \\
& \left(V_{z}\right)_{\eta=\frac{\pi}{2}}=u\left[1-\left\{\frac{2 \ln 2\left(\frac{a}{\varrho}\right)-1}{2 \ln 2\left(\frac{a}{b}\right)-1}\right\}\right] \quad[7]
\end{aligned}
$$

$$
\varrho \gg a \quad a \gg b
$$

Equations [6] and [7] give the stream velocity at different radial positions on the symmetry

Table 1. Velocity along symmetry axis (in streaming direction) $\left(V_{z}\right)_{n=0}$

\begin{tabular}{lll}
\hline Distance from tip & \multicolumn{2}{c}{ Velocity as a fraction of $u$} \\
\hline & Ellipsoid 1 & Ellipsoid 2 \\
& $a=10^{-4} \mathrm{~cm}$ & $a=1 \mathrm{~cm}$ \\
$0 \mathrm{~cm}$ & $b=\mathbf{1 0}^{-6} \mathrm{~cm}$ & $b=10^{-6} \mathrm{~cm}$ \\
$10^{-7}$ & 0 & 0 \\
$10^{-6}$ & 0.21 & 0.41 \\
$10^{-5}$ & 0.45 & 0.49 \\
$10^{-4}$ & 0.68 & 0.57 \\
$10^{-3}$ & 0.91 & 0.65 \\
$10^{-2}$ & 0.98 & 0.73 \\
\hline
\end{tabular}

Velocity along symmetry plane (in streaming direction) $\left(V_{z}\right)_{n=\frac{\pi}{2}}$

\begin{tabular}{clll}
\hline Distance from side surface & Radius $\varrho$ & \multicolumn{2}{c}{ Velocity as fraction of $u$} \\
\hline & & Ellipsoid 1 & \\
& & $a=10^{-4} \mathrm{~cm}$ & Ellipsoid 2 \\
$0 \mathrm{~cm}$ & $10^{-6} \mathrm{~cm}$ & $b=10^{-6} \mathrm{~cm}$ & $a=1 \mathrm{~cm}$ \\
$10^{-7}$ & 1.1 & 0 & $b=10^{-6} \mathrm{~cm}$ \\
$10^{-6}$ & 2.0 & 0.023 & 0 \\
$10^{-5}$ & $10^{-6}$ & 0.145 & 0.007 \\
$10^{-4}$ & $10^{-5}$ & 0.480 & 0.049 \\
$10^{-3}$ & $10^{-4}$ & 0.815 & 0.16 \\
$10^{-2}$ & $10^{-3}$ & 0.98 & 0.33 \\
\end{tabular}


plane. The results for ellipsoids where $a=1$ and $10^{-4} \mathrm{~cm}, b=10^{-6} \mathrm{~cm}$ is also given in Table 1.

\section{Discussion of Results}

From Table 1 it becomes immediately apparent that at both the side surface and the tip of the ellipsoids appreciable velocity changes occur over very small distances. This means the velocity gradients $\dot{\gamma}$ are high, typically of the order $10^{5} \mathrm{sec}^{-1}$ or higher, which in turn means that externally applied velocity gradients will have little significance in comparison to the local velocity gradients generated by the fibre.

We note that for both ellipsoids examined the velocity changes are of comparable magnitude (within a factor of ten) thus for simplicity, in the discussion to follow we will only consider the velocity values obtained for the ellipsoid where $a=10^{-4} \mathrm{~cm}$.

Examination of the velocity change on the symmetry axis shows that within IOA of the tip the stream velocity has reached 0.21 of its terminal value. Thus for a terminal velocity of $10 \mathrm{~cm} / \mathrm{sec}$ the local velocity gradient is $2.10^{7} \mathrm{sec}^{-1}$. With increasing distance from the tip the velocity gradient steadily decreases although it is still significantly large up to distances $\sim 10^{-4} \mathrm{~cm}$. It is recalled that this velocity gradient along the symmetry axis is purely longitudinal.

Because of the high aspect ratio of the ellipsoid considered $\frac{a}{b}=10^{2}$ it is justifiable to say that the radial velocity change along the symmetry plane is representative of radial velocity changes along the whole fibre with the exceptions of regions near the tip.

A comparison of the magnitude of the transverse velocity gradient on the side surface with the magnitude of the longitudinal velocity gradient on the symmetry axis is instructive. IO $\AA$ away from the side surface the velocity has reached 0.023 of its terminal velocity, a factor of ten, less than that on the symmetry axis. With increasing radial distance the transverse velocity gradient becomes weaker in a similar way to the decrease in longitudinal velocity gradient along the symmetry axis.
The results will now be discussed in molecular terms relating to the growth of shish kebab backbones. We see that immediately downstream of the tip for a stream velocity of $10 \mathrm{~cm} / \mathrm{sec}$ a longitudinal velocity gradient of magnitude $10^{5}-10^{7} \mathrm{sec}^{-1}$ is present, which both by theoretical estimates $(5,10)$ and experimental observation (6) could completely align molecules even of moderate length whether they are attached or not to the fibre.

Concerning molecular attachment; in spite of the fact that the shish kebab structures shown in Fig. 1 have been thoroughly washed with fresh solvent at the crystallization temperature of $103^{\circ} \mathrm{C}$, some intrinsic platelet overgrowth still remains attached to the backbone. This implies that at least some of the backbone and platelets are molecularly connected, (a conclusion also reached in other papers $(6,11))$. From this we conclude that part of a molecule can become attached on the surface or trapped within the crystal backbone for appreciable times leaving the remaining part of the molecule dangling freely in solution, where later, if quiescent conditions permit, it can take part in the crystallization of the molecularly connected platelet overgrowth.

Thus making the assumption that part of individual molecules can become attached or trapped to the crystal for significant times it is readily seen that molecules attached near the tip will have their free ends immediately extended along the fibre axis by the flow field. If there are sufficient molecules in this state and the supercooling is sufficiently large, the molecules will come into crystallographic register as extended chains, thereby offering a mechanism for chain extended crystal growth.

The question as to what happens in molecular terms on the side surfaces of the crystal is rather more complex and uncertain. By the fact that we observe backbone crystals up to $200 \AA$ diameter we conclude that the crystal can grow up to this dimension but no further. The factor which prevents further lateral growth of the extended chain backbone is at present unknown. Two possibilities will be considered, one of which is related to the nature of the flow field on the side surface. 
Firstly considering unattached molecules. As these molecules flow in the neighbourhood of the fibre side surface they will experience a transverse velocity gradient of magnitude $\sim 10^{4} \mathrm{sec}^{-1}$ up to ten times less than the extensional velocity gradients at the fibre tip. Theoretically (6) transverse velocity gradients even of this magnitude will be unable to extend the flexible molecules appreciably, thus the molecule will remain unaffected by the flow field.

If part of the molecule was attached to the fibre the unattached part of the molecule would be stretched in the stream direction. However as the stretched molecule approaches the side surface of the crystal, it will be coming into an increasingly weaker velocity gradient along its stretching axis due to the velocity being zero along the whole side surface of the crystal. In fact a molecule lying parallel to and on the side surface would experience a zero velocity gradient along its axis. In this situation the molecule will try to return to its entropically favoured random coil configuration; thus a possible mechanism is seen which prevents molecules lying down parallel to the crystal face and presumably crystallizing as extended chains; consequently lateral growth is prevented and can proceed only at the tip.

Alternatively Professor F. C. Frank made a further suggestion, namely that the chain ends of molecules may be excluded from the extended chain crystal, in which case the ends will somehow have to find their way to the side surface of the crystal. The concentration of chain ends will increase as the square of the radius of the fibre, whilst the area available at the crystal surface will only increase as the first power of the radius. Thus a limit will be reached at a critical radius when no more chain ends can be accommodated at the surface.

\section{Conclusions}

In spite of uncertainties concerning the limitation of lateral growth of the shish kebab backbone this paper has predicted that local velocity. gradients around anchored shish kebab fibres must dominate their hydrodynamic growth process. In addition the magnitude and nature of the velocity gradient at the tip will give optimum conditions for chain extension, and presumably oriented chain extended crystallization. This strongly suggests that the local velocity gradient at the tip is the controlling mechanism by which chain extended shish kebab backbones grow.

\section{Acknowledgements}

I would like to thank Professors $L$. Howarth, $F$. C. Frank and $A$. Keller, also Dr. D. T. Grubb and Dr. $D$. Peregrine for useful discussions on various aspects of this paper. I am also grateful to Messr. I.C.I. Ltt. for their financial support.

\section{Summary}

Hydrodynamic factors affecting the growth of anchored 'shish kebab' type fibrous crystals of polyethylene are examined. The problem is reduced to its simplest form of uniform streaming parallel to the major axis of a high aspect ratio ellipsoid. The Stokes flow solution shows that local velocity gradients near the tip and the side surface are very much greater than typically applied external velocity gradients; also the magnitude and nature of the velocity gradient at the tip strongly suggests a controlling mechanism by which the chain extended backbone of the fibre forms.

\section{Zusammenfassung}

Untersuchungsgegenstand sind die hydrodynamischen Faktoren, die das Wachstum von verankerten faserigen Polyäthylen-Kristallen des Typs "shish kebab" beeinflussen. Das Problem ist auf seine einfachste Erscheinungsform reduziert, nämlich auf gleichförmige Strömungen parallel zur Hauptachse eines Ellipsoids mit hoher Exzentrizität. Die "Stokes"Lösung zeigt, da $B^{\prime}$ das örtliche Geschwindigkeitsgefälle in der Nähe der Enden und der seitlichen Oberfläche viel stärker ist, als das typischerweise für ein äußeres Geschwindigkeitsgefälle gilt; ebenso verweisen Größe und Natur des Geschwindigkeitsgefälles an den Enden ganz deutlich auf einen Kontrollmechanismus, durch den sich das extended chain -Gerüst der wachsenden Faser, ihr zentraler Kern ausbildet.

\section{References}

1) Pennings, A. J., A. Zwijnenburg and R. Lageveen. Kolloid-Z. u. Z. Polymere. 25I, 500-501 (1973).

2) Pennings, A.J. and A. M. Kiel, Kolloid-Z. u. Z. Polymere 205, 160-162 (1965).

3) Pennings, A. J., J. M. A. A. van der Mark and H. C. Booij, Kolloid-Z. u. Z. Polymere 236, 99-111 (1970).

4) Frank, F. C., A. Keller and M. R. Mackley, Polymer 12, 7 467-473 (1971).

5) Hlavacek, B. and F. A. Seyer. Kolloid-Z. u. Z. Polymere 243, 32-41 (1971).

6) Markley, $M . R$. and A. Keller, Phil. Trans. Royal. Soc. (Lon). 278, 1276, 29-66 (1975). 
7) Pennings, A. J., J.M.A. A. van der Mark and A. M. Kiel, Kolloid-Z. u. Z. Polymere 237, 336 (1970).

8) Einstein, A., Annalen der Physik (4) 371-381 (1906).

9) Happel, $J$. and H. Brenner 'Low Reynolds' number hydrodynamics'. 96, 156, 509-511. (1965).

10) Peterlin, A., J. Pol. Sci. B. 4, $287-290$ (1966).

11) McHugh, A. J. and J. M. Scbultz. Kolloid-Z.

u. Z. Polymere 251, 193-213 (1973).
Author's address:

M. R. Mackley

Physics Laboratory

University of Bristol

Bristol BS8 ITL

United Kingdom 